\title{
Prediction of multipactor in the iris region of rf deflecting mode cavities
}

\author{
G. Burt and A. C. Dexter \\ Lancaster University, Engineering Department, Lancaster, LA1 4YR, United Kingdom
}

(Received 4 October 2011; published 13 December 2011)

\begin{abstract}
Multipactor is a major cause of field limitation in many superconducting rf cavities. Multipacting is a particular issue for deflecting mode cavities as the typical behavior is not well studied, understood, or parametrized. In this paper an approximate analytical model for the prediction of multipactor in the iris region of deflecting mode cavities is developed. This new but simple model yields a clear explanation on the broad range of rf field levels over which the multipactor can occur. The principle multipactors under investigation here are two-point multipactors associated with cyclotron motion in the cavity's rf magnetic field. The predictions from the model are compared to numerical simulations and good agreement is obtained. The results are also compared to experimental results previously reported by KEK and are also found in good agreement.
\end{abstract}

DOI: 10.1103/PhysRevSTAB.14.122002

PACS numbers: 52.20.Dq, 29.20.-c, 52.35.Hr, 29.27.Bd

\section{INTRODUCTION}

A major limitation to the gradient of many rf cavities is multipactor. Multipactor is a resonant electron phenomenon where electrons following specific trajectories become synchronized to the operating mode's frequency and then multiply to give an unacceptable current [1]. If an electron strikes a surface of an rf cavity and creates a secondary electron, that electron can then get accelerated away from the surface by the rf electric field; it can then follow a complicated trajectory in the electromagnetic field of the cavity until it strikes a surface, possibly creating another secondary electron. Secondary electron production depends on the impact velocity of returning electrons and is normally greater than one for an impact energy range of several hundred $\mathrm{eV}$ above a few tens of $\mathrm{eV}$ for most materials [2]. Where secondary electron trajectories return to their approximate starting position and phase within a finite number of secondary emission steps, and the total number of electrons following that trajectory is increasing then multipactor is said to occur. As the electrons multiply they absorb power from the rf field. If the power input is limited the multipactor often reaches equilibrium before a catastrophic vacuum arc occurs. During this process the rf field levels where the multipactor commenced becomes reduced and multipactor trajectories become less likely. The multipactor discharge can also self-limit when space charge fields modify and dilute the phase space for resonant trajectories [3]. At equilibrium the cavity maintains a constant rf field and, hence, accelerating gradient and the number of electrons remains roughly constant.

Published by the American Physical Society under the terms of the Creative Commons Attribution 3.0 License. Further distribution of this work must maintain attribution to the author(s) and the published article's title, journal citation, and DOI.
Multipactor has been well studied in rectangular waveguides [4], circular waveguides [5], coaxial lines [6], and accelerating cavities [7]. In accelerating cavities it has been established that an elliptical cavity geometry causes any electrons to travel towards the location of maximum magnetic field and minimum electric field at the top of the cavity equator. The electrons make semicircular arcs across the cavity with decreasing radii for each generation. As smaller arcs occur in regions of smaller electric field, eventually the electrons will not have sufficient energy to create enough secondary electrons to maintain multipactor.

There has been little study of multipactor in transverse deflecting cavities. Transverse deflecting cavities, and a subset of these known as crab cavities, are required for a number of applications in particle accelerators where the bunches need to be rotated with respect to the direction of motion (also known as crabbed) [8] or kicked [9]. These cavities use a dipole mode for their operation (where the field has a $360^{\circ}$ variation around the cavity azimuthally) as opposed to a monopole mode which is normally used in accelerating cavities. The fundamental dipole mode of a pillbox cavity (normally a $\mathrm{TM}_{110}$ mode) has a transverse magnetic field on the cavity longitudinal axis of symmetry providing the transverse kick. However, beam pipes must be added to the cavity in order to allow the beam to enter and exit the cavity, and for multicell cavities the cells will be coupled via irises. The $\mathrm{TM}_{110}$ mode of the cavity will couple to the cutoff $\mathrm{TE}_{11}$ waveguide mode of the iris and/or beam pipe altering the fields in this region. This creates a hybrid mode in the cavity [10].

The KEKB crab cavity was found to have a multipacting band in the iris region around a location where the electric field was zero and the magnetic field was maximum [11]. A large temperature rise was found in this location that grew as the field increased and then disappeared at a peak surface field of $20 \mathrm{MV} / \mathrm{m}$. Temperature spikes were found at peak surface electric fields of 12 and $18 \mathrm{MV} / \mathrm{m}$. This 
was suspected to be multipactor related to the cyclotron motion of electrons in the strong magnetic field. The $12 \mathrm{MV} / \mathrm{m}$ field was assumed to be a one-point multipactor and the $18 \mathrm{MV} / \mathrm{m}$ was predicted to be a two-point multipactor. Simple particle tracking calculations implied that the two-point multipactor had an electron trajectory that made semicircular arcs centered on the electric field null as described above. Numerical simulation has predicted multipactor in the iris of many dipole cavities operating at frequencies below $1 \mathrm{GHz}$ [12]

In the design of an $800 \mathrm{MHz}$ two cell elliptical crab cavity for the Large Hadron Collider (LHC), it was found via numerical modeling that most of the potential designs exhibited a first order multipactor in the iris region. Study of this multipactor in CST PARTICLE STUDIO [13], detailed later in this paper, has shown this to be a two-point multipactor, where the electrons follow semicircular trajectories traveling around the peak magnetic field location. The simulations suggest that the multipactor occurs around the location of the maximum magnetic field and the minimum electric field on the iris similar to the KEKB crab cavity. This type of multipactor is dissimilar to the multipactor found in the equators of accelerating cavities [7] where the electrons orbit around the electric field null. In the accelerating cavities the equator can be shaped to cause the radius of orbit to decrease until the electrons no longer have enough energy to create secondary electrons. This paper shows that for deflecting and crab cavities, however, the radius of the electron trajectories stays finite and there are orbits which maintain synchronism with the rf leading to multipactor. In order to understand this phenomenon an approximate analytical model has been developed. A key advance made by this paper is the identification of a relatively simple relationship between the phase of the multipactor and the magnetic field and also the emission velocity and the equilibrium orbit radius. With this new understanding, one can quickly dismiss many cavity designs that will not work. In this paper the approximate analytical model is validated against highly accurate tracking simulations in the fields of the $\mathrm{HM}_{11}$ mode and also CST PARTICLE STUDIO simulations of multipactor in dipole cavities.

Section II of this paper describes a useful field approximation for dipole deflecting cavities that is assumed for trajectory calculations. Section III derives an approximate analytical formula that predicts the occurrence of multipactor trajectories in dipole deflecting cavities without the requirement of a full solution of the equations of motion, and this is compared to numerical tracking simulations utilizing the assumed electromagnetic field profile in Sec. IV. Section V considers the effect of a distribution in the electron emission velocities in both the analytical and numerical calculations and this is compared to the KEKB crab cavity multipactor data in Sec. VI. In Sec. VII higher orders of multipactor are considered.
Finally, in the last section we undertake numerical tracking simulations in the electromagnetic fields calculated in CST PARTICLE STUDIO to validate the approximate formula.

\section{FIELDS IN THE IRIS REGION OF RF DEFLECTING CAVITIES}

Dipole cavities of the type commonly used in particle accelerators usually operate in modes similar to the $\mathrm{TM}_{110}$ mode of a pillbox cavity. Because of the beam pipes and iris, used on these cavities to allow the beam to enter and exit the cavity, this mode will couple to the fundamental dipole mode of the beam pipe which is the $\mathrm{TE}_{11}$ mode. This coupling creates a hybrid mode which has fields in the iris/ beam pipe region similar to the $\mathrm{HM}_{11}$ mode [10]. In order to understand the multipactor found in $\mathrm{rf}$ deflecting cavities, we must investigate the interaction between an electron and the fields of this mode. If the cavity is to be utilized on a relativistic beam then it is necessary for the phase velocity of the operating mode to be equal to the speed of light in a vacuum, in this case the fields of the $\mathrm{HM}_{11}$ mode for a standing wave cavity are simplified and can be approximated as [14]

$$
\begin{gathered}
E_{x}=E_{o} \frac{k}{4 a}\left(a^{2}-y^{2}+x^{2}\right) \cos \left(k_{z} z\right) \cos (\omega t) \\
E_{y}=E_{o} \frac{k}{2 a} x y \cos \left(k_{z} z\right) \cos (\omega t) \\
E_{z}=-E_{o} \frac{x}{a} \sin \left(k_{z} z\right) \cos (\omega t) \\
c B_{x}=E_{o} \frac{k}{2 a} x y \sin \left(k_{z} z\right) \sin (\omega t) \\
c B_{y}=-E_{o} \frac{k}{4 a}\left\{k\left(a^{2}-y^{2}+x^{2}\right)-\frac{4}{k}\right\} \cos \left(k_{z} z\right) \sin (\omega t) \\
c B_{z}=E_{o} \frac{y}{a} \cos \left(k_{z} z\right) \sin (\omega t),
\end{gathered}
$$

where $E_{0}$ is the electric field amplitude, $k=\omega / c$ is the wave number, and $k z$ is the wave number in the axial direction, $\omega_{\mathrm{rf}}$ is the $\mathrm{rf}$ angular frequency, and $a$ is the iris radius. As we are interested in trajectories that are circular, centered around the electric node of the cavity wall, it is more useful to express these fields in cylindrical coordinates with the origin at the center of the electron orbit, which occurs at $(x=0, y=a)$. If we consider the fields at the middle of an iris $(z=0)$, then the standing wave will only have transverse electric field components and a longitudinal magnetic field component on this plane. The approximate fields expanded on the plane and about this the point on the iris wall in polar coordinates is given as

$$
E_{\varphi}=-E_{o} \frac{k}{4 a}\left(2 r a-r^{2} \sin \varphi\right) \cos (\omega t)
$$




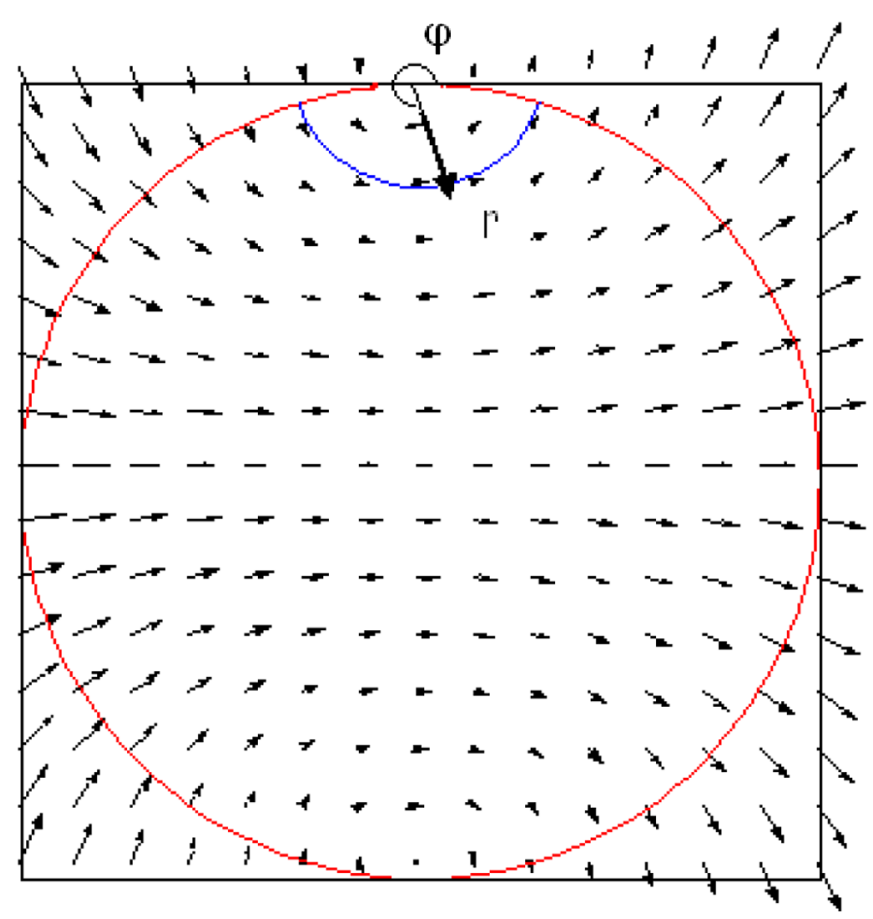

FIG. 1. $\mathrm{HM}_{11}$ mode transverse electric field determined from (7) and (8) with iris walls shown in red and example electron trajectory in blue.

$$
\begin{aligned}
E_{r} & =-E_{o} \frac{k}{4 a} r^{2} \cos \varphi \cos (\omega t) \\
c B_{z} & =\frac{E_{o}}{a}(a-r \sin \varphi) \sin (\omega t),
\end{aligned}
$$

where $r$ is the radius from the origin located at the waveguide wall $(x=0, y=a)$, and $\phi$ is the azimuthal angle around the waveguide. Hence at small radii, when $r \ll a$, the electric field has an azimuthal pattern that is roughly constant at constant radius, and the magnetic field is maximum, as can be seen in Fig. 1 .

\section{ELECTRON TRAJECTORIES IN HYBRID MODES}

For multipactor to occur, the average number of secondary electrons produced per electron impact with the surface should be above unity. For most materials used in accelerators, including niobium, the secondary electron emission yield, $\delta$, is only above unity at low electron impact energy (typically 40-1000 eV for niobium) [15]. This often means that multipactor occurs at low electric fields and hence electron velocities; consequently, any magnetic field, unless very strong, will have little effect on multipactoring electron trajectories as the cyclotron radius becomes very large compared to the structure geometry. For dipole deflecting cavities, known multipactor trajectories are in a location of strong magnetic field but weak electric field in the cavity iris or beam pipe [11]. When electrons travel in a strong (static) magnetic field with a weak crossed (static) electric field they undergo epicycloidal motion [16]. For a standing wave rf cavity, the electric and magnetic fields oscillate and are out of phase. This promotes backwards and forwards motion on curved arcs rather than motion on an epicycloid. The nonrelativistic equations of motion for an electron moving in the $r \phi$ plane under the influence of a $z$ directed magnetic field are

$$
\begin{aligned}
\ddot{r}-r \dot{\varphi}^{2} & =-\frac{e}{m}\left(E_{r}+r \dot{\varphi} B_{z}\right) \\
2 \dot{r} \dot{\varphi}+r \ddot{\varphi} & =-\frac{e}{m}\left(E_{\varphi}-\dot{r} B_{z}\right),
\end{aligned}
$$

where $e$ is the charge of an electron and $m$ is the rest mass of the electron. The right-hand sides of these equations involving electric and magnetic fields takes a simple form on the $z$ plane through the iris of a dipole cavity. Near the electric field null when $r \ll a$ powers of $r^{2}$ in (7) and (8) can be neglected and the $r$ independent term in (9) dominates; hence, a suitable approximation for the field in this region is that

$$
\begin{aligned}
& E_{r}=0 \\
& E_{\varphi} \approx-E_{o} \frac{k r}{2} \cos (\omega t) \\
& B_{z} \approx \frac{E_{o}}{c} \sin (\omega t) .
\end{aligned}
$$

Making definition

$$
B_{o}=\frac{E_{o}}{c}
$$

and inserting (12b) and (13) into (11)

$$
2 \dot{r} \dot{\varphi}+r \ddot{\varphi}=\frac{e B_{o}}{2 m}[\omega r \cos (\omega t)+2 \dot{r} \sin (\omega t)] .
$$

This can be written as

$$
\frac{1}{r} \frac{d\left(r^{2} \dot{\varphi}\right)}{d t}=\frac{e B_{o}}{2 m} \frac{1}{r} \frac{d}{d t}\left[r^{2} \sin (\omega t)\right]
$$

As there are no terms in $r$ outside the differentiations we can integrate to get

$$
r^{2} \dot{\varphi}=\frac{e B_{o}}{2 m} r^{2} \sin (\omega t)+C,
$$

where $C$ is a constant set by the initial (angular) velocity. Assuming that at $t=t_{0}, r=r_{i}$ and the initial velocity $v_{i}=r_{i} \omega$ is tangential, then from (15)

$$
\dot{\varphi}=\frac{e}{2 m} B_{o} \sin (\omega t)+\frac{1}{r^{2}}\left\{r_{i} v_{i}-\frac{e}{2 m} r_{i}^{2} B_{o} \sin \left(\omega t_{0}\right)\right\}
$$

With the approximate fields of (12) and (13) and first equation of motion (10) becomes 


$$
\ddot{r}-r \dot{\varphi}^{2}=\frac{e}{m} B_{o} r \dot{\varphi} \sin (\omega t) .
$$

Inserting (16) into (17) and applying some algebraic manipulation yields

$\ddot{r}+\left(\frac{e}{2 m}\right)^{2} B_{o}^{2} r \sin ^{2}(\omega t)-\frac{1}{r^{3}}\left\{r_{i} v_{i}-\frac{e}{2 m} r_{i}^{2} B_{o} \sin \left(\omega t_{0}\right)\right\}^{2}=0$.

This equation can be numerically integrated very quickly to give the orbit radius as a function of time given the starting radius $r_{i}$, the starting time $t_{0}$, and the launch velocity $v_{i}$. Given the radius at any time, Eq. (16) can be integrated numerically to give the angular position at any time. For rf field values of interest, Eqs. (16) and (18) yield orbits that are close to being semicircles and converge to a specific radius.

A synchronous multipactor orbit must take half an $\mathrm{rf}$ period; hence, if it starts at $t_{0}$ it must finish at $t_{0}+T / 2$, where $T$ is the rf period. Integrating (16) in time between $t_{0}$ and $t_{0}+\pi / \omega$ for a $180^{\circ}$ arc from $\phi=0$ to $\phi=\pi$ gives

$$
\begin{aligned}
\pi= & -\frac{e B_{o}}{2 m \omega}\left\{\cos \left(\omega t_{0}+\pi\right)-\cos \left(\omega t_{0}\right)\right\} \\
& +\left\{r_{i} v_{i}-\frac{e}{2 m} r_{i}^{2} B_{o} \sin \left(\omega t_{0}\right)\right\} \int_{t_{0}}^{t_{0}+\pi / \omega} \frac{d t}{r^{2}} .
\end{aligned}
$$

For a synchronous orbit the final radius is equal to the initial radius and numerical simulation shows that the variation of the radius is small. On this basis we can approximate the integral as

$$
\int_{t_{0}}^{t_{0}+\pi / \omega} \frac{d t}{r^{2}} \approx \frac{\pi}{\omega r_{i}^{2}}
$$

hence near a synchronous orbit (19) becomes

$$
\pi \approx \frac{e}{m \omega} B_{o} \cos \left(\omega t_{0}\right)+\frac{\pi v_{i}}{\omega r_{i}}-\frac{\pi e}{2 m \omega} B_{o} \sin \left(\omega t_{0}\right) .
$$

Finally, this can be rearranged to give an approximate expression for the magnetic field as a function of starting phase, orbit radius, and initial velocity for a synchronous multipactor orbit:

$$
B_{o} \approx \frac{2 \pi m}{e} \frac{\left(\omega-v_{i} / r_{i}\right)}{2 \cos (\omega T)-\pi \sin \left(\omega t_{0}\right)} .
$$

In order to use (20), one needs to know the final orbit radius and this requires a numerical solution of (18). Remarkably there seems to be a very simple formula that predicts the final orbit radius and the starting phase for a known rf field. We will see later the multipactor orbit radius is almost linear with initial velocity; hence, in practice Eq. (20) does not have a significant dependency on $r_{i}$.

Looking back at (17) if the electrons start from rest, then initially $\dot{\varphi}=0$ and hence $\ddot{r}=0$. In reality the electrons will have a small initial velocity which is investigated later.
The electric field will accelerate the electrons and $\dot{\varphi}$ will start to increase. If an electron is launched at a time such that $\omega t \sim 0$ when the magnetic field is small then both the magnetic field and the electron angular velocity will increase approximately linearly with time and the magnetic field acts to suppress $\ddot{r}$ by curving the electronic path. However, if the electrons are launched at a positive phase, the electrons are decelerated and their direction is reversed before impacting on the other side such that multipactor is unlikely. If an electron is launched at a time such that $-0.5 \pi<\omega_{\mathrm{rf}} t<0$ (negative phase) then the magnetic field is initially negative, and $\ddot{r}$ will increase; however, as the electron velocity starts low the electron does not travel far before the magnetic field becomes positive. As the velocity increases, the magnetic field increases causing the electrons to move in roughly circular orbits. Interestingly, orbits in the rf field phase focus such that the starting radius eventually equals the arrival radius. This means that the net contribution of $\ddot{r}$ has virtually no effect on the electron transit time between two points on the iris surface. Neglecting the radial acceleration in (17) gives

$$
\dot{\varphi}=\frac{e}{m} B_{o} \sin (\omega t) \text {. }
$$

This is still consistent with (16) for trajectories that have a low $\ddot{r}$, as the radius is linearly dependent on the starting electron velocity; hence, the last term in Eq. (16) is not truly dependent on starting velocity, hence this term can, and does, account for the factor of $1 / 2$ in the first term as both terms are roughly equal. For first order multipactor to occur between two points on the cavity iris then the electrons must rotate by 180 degrees about the electric field null in one half period. If the orbit is semicircular and centered on the electric field null then the resonant frequency can be obtained using this condition by integration of Eq. (21).

For the case where the starting magnetic field is negative, Eq. (21) is still valid and can be used to calculate transit time; however, the effective center of the orbit moves away from the field null. This is because electrons start curling in the opposite sense to their subsequent motion moving to a different orbit from $\mathrm{A}$ to $\mathrm{B}$ as shown in Fig. 2.

Consequently, the electrons must now rotate around an angle slightly larger than 180 about the new center point $C$. We can find the angle the electrons move around by integrating Eq. (21) only from the point where the magnetic field is positive, when the phase is zero, to the phase where it impacts the wall, as the electron motion is small when the magnetic field is negative, hence,

$$
\Delta \varphi=\int_{0}^{\pi+\phi} \frac{e B_{o}}{m \omega} \sin [\theta] d \theta
$$

where $\varphi$ is the starting rf phase. The extra physical angle the electrons must rotate, $\phi_{\text {neg }}$, due to electron movement to point $B$ while the magnetic field is negative, is proportional 


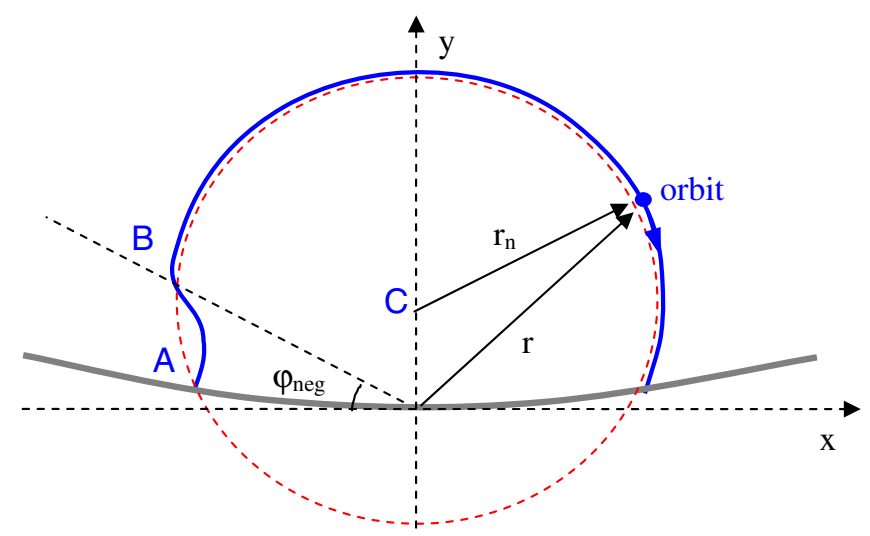

FIG. 2. A typical trajectory showing the orbit center $C$ and $\phi_{\text {neg. }}$. The iris curvature has been exaggerated for illustrative purposes.

to the starting phase. As the electrons must rotate through an angle of 180 degrees plus $\phi_{\text {neg }}$ in one half rf cycle we can rearrange this to

$$
B_{o}=\frac{\omega m\left(\pi-\varphi_{\mathrm{neg}}\right)}{e(1+\cos [\phi])} .
$$

This is only dependent on the starting rf phase and the rf frequency, plus $\phi_{\text {neg }}$ which we will determine empirically later from numerical simulations, hence this formula is suitable for predicting multipactor. For a launch phase of zero, when $\phi_{\text {neg }}=0$, we obtain the lowest magnetic field where first order multipactor can occur:

$$
B_{o}=\frac{\omega m \pi}{2 e} .
$$

Hence, for a given resonant frequency the multipactor will start at the same peak magnetic field.

As most crab cavities operate at a peak surface magnetic field of up to $100 \mathrm{mT}$, we can see from Eq. (24) that first order multipactor is unlikely to occur in crab cavities operating at frequencies above $1.78 \mathrm{GHz}$. This is confirmed by the lack of multipactor found in the FNAL/ILC $3.9 \mathrm{GHz}$ crab cavities [17]. However, higher order multipactor could still occur in higher frequency cavities, and this will be discussed later.

\section{NUMERICAL TRACKING SIMULATIONS AND COMPARISON WITH THEORY}

In order to investigate multipactor in the iris of deflecting and crab cavities, large numbers of simulations were performed using a high precision numerical tracking code. The tracking code numerically integrates the relativistic equations of motion with a fourth order Runge-Kutta algorithm using a variable time step reset on each iteration in accordance with the electronic velocity and the anticipated acceleration to ensure accuracy at the micrometer scale. The field profile of the $\mathrm{HM}_{11}$ mode in a $70 \mathrm{~mm}$ radius circular waveguide was calculated from the analytical expressions, (1)-(6). Electron trajectories from various emission points and at various launch phases were calculated by simulating the motion of a single macroparticle that did not perturb the predefined electromagnetic field in the cavity. A variable charge was assigned to the single macroparticle to carry information on the multipactor multiplication process. The initial energy of the macroparticle was set to $4 \mathrm{eV}$ for most of the simulations performed within this paper except those where the initial energy/velocity was swept. At each impact the charge of the secondary macroparticle is set depending on the primary's impact energy and the secondary emission yield (SEY). The macrosecondary is then tracked in the fields to the next impact where the yield calculation is repeated. This continues either until the charge drops below $90 \%$ of the initial charge, or rises above a value implying at least 20 surface interactions at the maximum yield or the calculation has persisted for $400 \mathrm{rf}$ cycles. The secondary particles were also set to have an initial energy of $4 \mathrm{eV}$.

This simple method can be used to identify resonant trajectories. Here we ignore the SEY curve of the material and focus on investigating if the macroparticle follows a resonant trajectory. If the electron is launched at a phase and position near to resonant conditions, phase focusing [1] causes the electrons to gradually move towards the resonant trajectory making the search for resonant trajectories much simpler. When a resonant trajectory is found for a given amplitude, the emission phase and position of the macroparticle is recorded.

As the initial interest in the multipactor was for an elliptical crab cavity for the LHC, a rf frequency of $800 \mathrm{MHz}$ and an iris radius of $70 \mathrm{~mm}$ were chosen for study. The trajectories of interest were those with semicircular trajectories with periods equal to half the rf period. Such a trajectory for a magnetic field of $54 \mathrm{mT}$ is shown in Fig. 3. These resonant trajectories were found to occur between peak rf magnetic field values of 41 and $60 \mathrm{mT}$, respectively corresponding to phases between 0 and -48 degrees. In this instance it should be pointed out that for $\mathrm{rf}$ magnetic fields below $44 \mathrm{mT}$ the impact energy would be too low to generate multipactor. A study of the numerical trajectories over a wide range of amplitudes and phases showed that the physical angle the electrons cover during the negative proportion of the magnetic fields is roughly equal to half the starting phase $\left(\phi_{\text {neg }}=-\varphi / 2\right)$. Inserting this relationship in (23) yields a modified form (25):

$$
B_{o}=\frac{\omega m(\pi+\phi / 2)}{e(1+\cos [\phi])} .
$$

The approximation that $\dot{r}=0$, and $\ddot{r}=0$ is checked as well. It was found that if we use the waveguide wall as the origin then the terms are nonzero as the negative magnetic field causes the center of the electron trajectory to be offset from the walls. However, if we choose the origin to be 


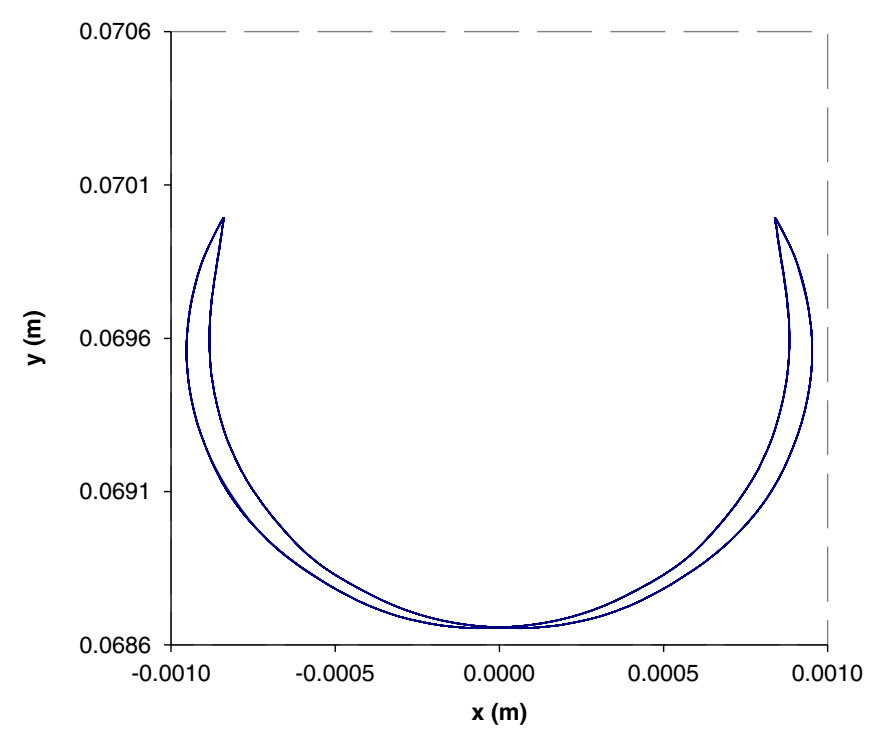

FIG. 3. An example of a resonant trajectory in the iris of a dipole cavity, calculated using the tracking code, for a $70 \mathrm{~mm}$ radius iris, rf frequency of $800 \mathrm{MHz}$, and a magnetic field of $54 \mathrm{mT}$.

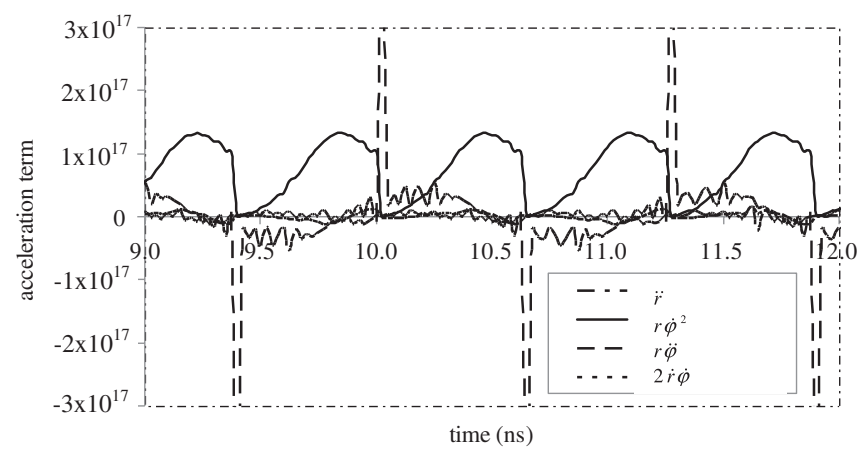

FIG. 4. Acceleration terms for a launch phase of -45 degrees where the radius is calculated from the center of the electron orbit.

offset from the wall by a small amount, at point $\mathrm{C}$ shown on Fig. 2, then $\dot{r}=0$, and $\ddot{r}=0$, as predicted. The four acceleration terms are shown in Fig. 4. This adds a small error to the equations as the fields are centered around a different point from the multipactor trajectories, however as the offset is small the effect should be negligible. For simplicity we will assume the distance between these two centers is small and neglect any terms related to the difference in the two centers.

Using this relation we can analytically calculate the relationship between magnetic field amplitude and starting phase for multipactor in deflecting mode cavity iris'. Using the $70 \mathrm{~mm}$ radius iris' used in the numerical calculations, for phases where multipactor was expected to occur ( 0 to -48 degrees), the analytical theory predicts multipactor at peak magnetic field values of 45 to $55 \mathrm{mT}$, as can be seen in Fig. 5, which is in good agreement with the

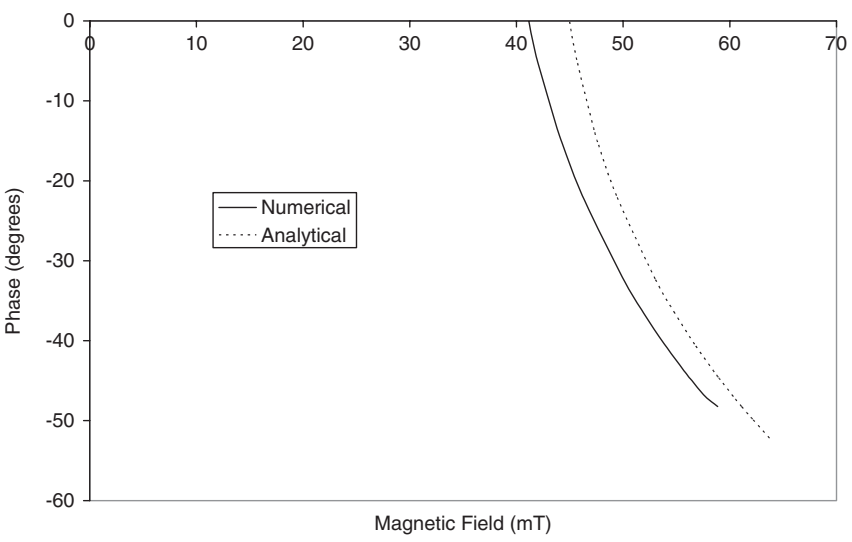

FIG. 5. Launch phase of resonant electron trajectories as a function of peak magnetic field for a $70 \mathrm{~mm}$ radius iris and $\mathrm{rf}$ frequency of $800 \mathrm{MHz}$.

numerical simulations. The analytical and numerical curves in Fig. 5 have the same trend but have a small DC offset between them and agree with each other to within $9 \%$.

\section{IMPACT VELOCITY/ENERGY OF ELECTRONS}

For multipactor to occur the secondary emission yield (SEY), for the electron impact energy of the trajectory, must be greater than one. For most materials this occurs at a finite range of impact energies, giving a finite range of phases and amplitudes where multipactor can occur. For niobium the SEY typically increases above unity between 40 and $1000 \mathrm{eV}$, although a high temperature bakeout at 350 deg $C$ can reduce this range down to 117 to $950 \mathrm{eV}$ [15]. The peak SEY occurs for niobium between 300$350 \mathrm{eV}$ and has a magnitude of around 1.6, although can vary between 1.2 and 2.3. Hence, it is important to know the impact energy of the electrons to predict whether multipactor will occur or not. In the absence of a radial velocity, hence constant radius, impact velocities are readily calculated from (11). The radial velocity is typically small and averages to zero; hence, as an approximation one can take $\dot{r}=0$ so that Eq. (11) becomes

$$
r \ddot{\varphi}=-\frac{e}{m} E_{\varphi} .
$$

The velocity of the electron can be given as an integral of (26) with respect to time plus the initial electron velocity. Launching the electron at phase $\varphi$ then the electron velocity can be given as

$$
\begin{aligned}
v & =r \dot{\varphi}=-\frac{e}{m} \int_{0}^{t_{1}} E_{\varphi} d t+v_{i}=-\frac{e}{m \omega} \int_{\phi}^{\theta_{1}} E_{\varphi} d \theta+v_{i} \\
& =v_{i}+\frac{e\left|E_{\varphi}\right|}{m \omega}\left[\sin \left(\theta_{1}\right)-\sin (\phi)\right],
\end{aligned}
$$

where $v_{i}$ is the initial emission velocity of the electrons. For first order multipactor the electrons will strike the 
surface after a half rf period (at phase $\theta_{1}=\pi+\varphi$ ), hence the impact velocity can be given by Eq. (27) as

$$
v_{\text {impact }}=v_{i}-\frac{2 e\left|E_{\varphi}\right|}{m \omega} \sin (\phi) .
$$

Inserting the equation for the azimuthal electric fields, (12b), into (21) gives

$$
v_{\text {impact }}=v_{i}-\frac{e r E_{0}}{m c} \sin (\phi) .
$$

This means that at a launch phase of $\varphi=0$ the impact energy would be equal to the initial energy, which is likely to be only a few $\mathrm{eV}$, hence multipactor can only occur at lower starting phases where the impact energy is large enough to have a SEY above one. However, in order to calculate the impact energy of the electrons, we must know the radius of the orbit in order to calculate the electric field strength, $E_{\varphi}$. We can find the radius of orbit by considering the distance the electron has to travel and the electron velocity. Equation (26) can be rewritten as

$$
s=r \varphi=\int v d t .
$$

The trajectories are only approximately circular. The numerical simulation show that the trajectories although roughly circular are not centered on the cavity surface. The center of the orbit moves away from the surface due to the motion of the electrons when the magnetic field is negative. Hence, in one half period we can combine (27) and (30) to give

$\left(\pi+2 \varphi_{\text {neg }}\right) r=\pi \frac{v_{i}}{\omega}+\frac{e\left|E_{\varphi}\right|}{m \omega^{2}} \int_{\phi}^{\pi+\phi}[\sin (\theta)-\sin (\phi)] d \theta$,

where $v_{i}$ is the initial electron velocity. Solving (31) and rearranging yields the predicted average radius of the electron orbit:

$$
r=\frac{2 \pi m c v_{i}}{\left\{\left(\pi+2 \varphi_{\text {neg }}\right) 2 m c \omega-e E_{0}[2 \cos (\phi)-\pi \sin (\phi)]\right\}} .
$$

For multipactor to be stable an electron is launched at an incorrect phase or position should be restored to the resonant phase and trajectory within a few rf cycles by the electromagnetic fields, known as phase focusing [1]. In order to study the phase focusing, the resonant phase and launch position for a $70 \mathrm{~mm}$ radius beam pipe was obtained for a peak surface magnetic field of $55.8 \mathrm{mT}$ and an initial energy of $4 \mathrm{eV}$. The electrons were then launched at the correct position $(x=1.22 \mathrm{~mm})$ but at the incorrect phase. The position and phase of the electrons was then recorded for each preceding impact. The impact position was not found to vary greatly, and the impact phase was quickly restored to the resonant phase (46.1 deg) within a single rf cycle, as can be seen in Fig. 6 .

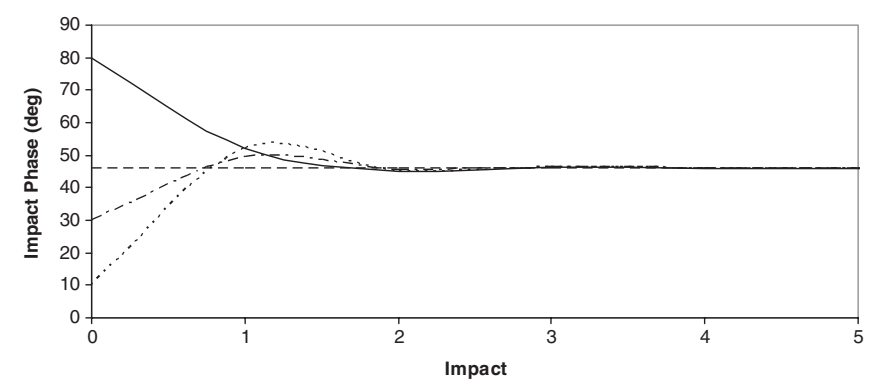

FIG. 6. Phase focusing of the electrons towards the resonant phase for electrons starting at the incorrect phase.

Next the electrons were launched at the correct phase but the incorrect position. The radius of the electron orbit was found to step towards the resonant trajectory within a few rf cycles as can be seen in Fig. 7.

Equation (32) shows how critical the initial electron emission energy is in determining the radius of orbit and, hence, the impact energy. Numerical simulations were performed where the initial electron velocity was fixed to a constant value for an iris radius of $70 \mathrm{~mm}$ and a $\mathrm{rf}$ frequency of $800 \mathrm{MHz}$. For a constant field level, the initial electron kinetic energy was swept between 0.5 to $5 \mathrm{eV}$ and the resonant trajectories were recorded, as seen in Figs. 8 and 9 . The starting phase was set to roughly -45 degrees and phase focusing was used to adjust this to the resonant launch phase. As can be seen the radius of orbit is almost linear with increasing initial electron velocity in excellent agreement with (25). In each case the resonant launch phase was not found to vary by more than 3 degrees due to phase focusing.

These trajectories had an average radius varying from $0.5 \mathrm{~mm}$ for the $44 \mathrm{mT}$ magnetic field to $3.6 \mathrm{~mm}$ at a

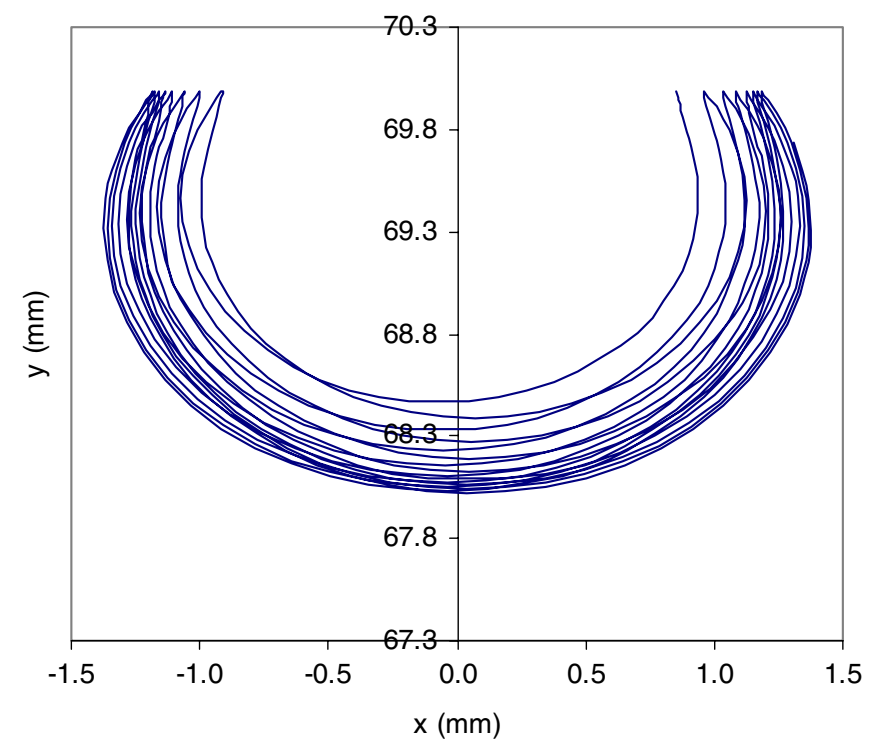

FIG. 7. Phase focusing of the electrons towards the resonant orbit. 


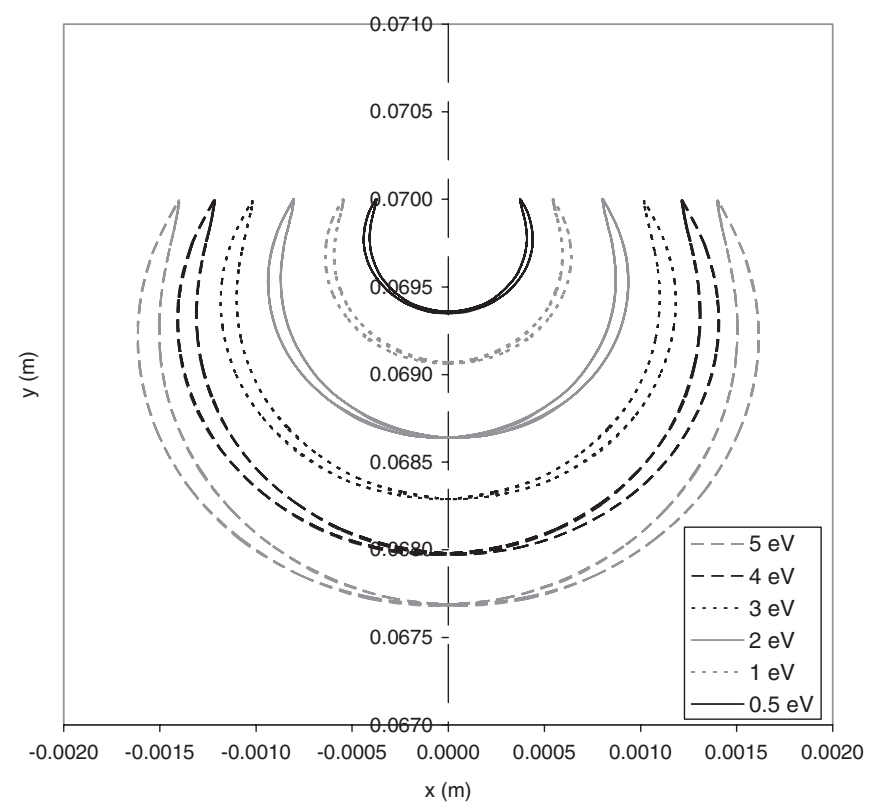

FIG. 8. Electron trajectories for various initial electron energies.

magnetic field of $58 \mathrm{mT}$. Utilizing the previous approximation that $\left(\phi_{\text {neg }}=-\varphi / 2\right)$, the analytical calculation predicted similar orbit radii, as can be seen in Fig. 10. This gives very good agreement with the numerical calculations.

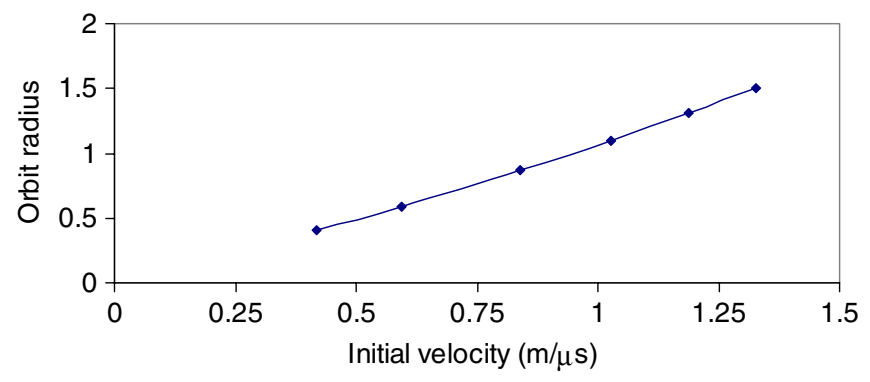

FIG. 9. Orbit radii as a function of initial electron velocity.

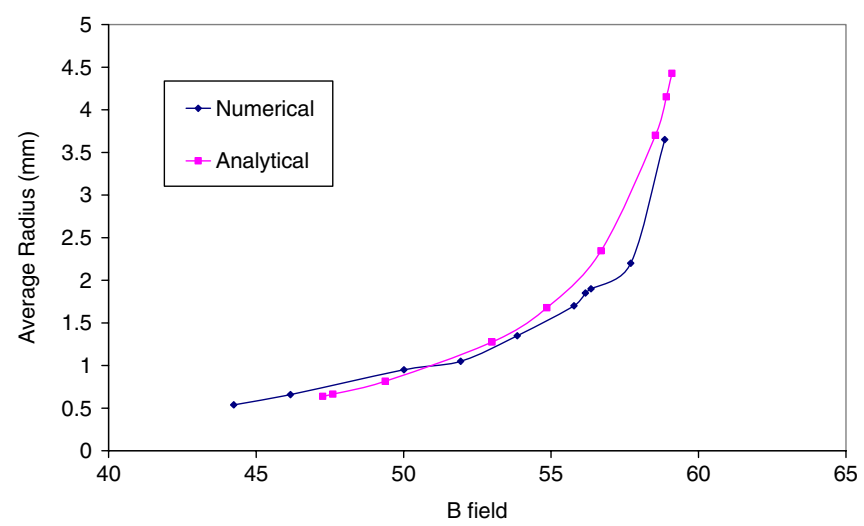

FIG. 10. Orbit radius against peak surface magnetic field calculated using tracking code and with analytical calculations.
The impact velocity can now be calculated by inserting (32) into (29) giving

$$
\begin{aligned}
& v_{\text {impact }} \\
& \quad=v_{i}\left(1-\frac{2 \pi e E_{0} \sin (\phi)}{\left\{\left(\pi+2 \varphi_{\text {neg }}\right) 2 m c \omega-e E_{0}[2 \cos (\phi)-\pi \sin (\phi)]\right\}}\right) .
\end{aligned}
$$

The electrons may start at a lower orbit and traverse backwards and forwards moving to a slightly larger orbit on each cycle creating more secondaries as it does so. This can continue for several cycles until the synchronous electrons reach an orbit where their impact energy becomes too high to produce multipactor. This may occur over several cycles and will produce a large increase in secondaries before it is extinguished.

Again it is noted the impact energy is linearly proportional to the initial electron energy. Numerical simulations were performed for fixed initial energy between 0.5 and $10 \mathrm{eV}$ and the impact energy is recorded, as found in Fig. 11. The numerical simulations confirm that the impact energy is proportional to initial energy.

The impact energy of these particles varies between 17 to $1420 \mathrm{eV}$ with the lower impact energy being at the lower phase. This is compared to the calculated impact energies of 17 to $3218 \mathrm{eV}$, which is in fair agreement with the numerical results. The high impact energy predicted for larger magnetic fields is due to the high sensitivity to the orbit radius. A comparison of the numerical and analytically calculated impact energies is shown in Fig. 12. The impact energy is very sensitive to the magnetic field and initial electron energy hence small errors, due to the approximations used previously, lead to a discrepancy at higher fields, when the starting phase is large and negative. If we assume a typical niobium sample with a SEY above 1.0 between 50 and $1000 \mathrm{eV}$, then multipactor will occur for magnetic fields between 48 and 56-58 mT.

It is noted that in the numerical simulations if a higher peak magnetic field is used the macroparticle will be close to resonance at higher impact phases. For this case the radius of the orbit will increase slightly in each half

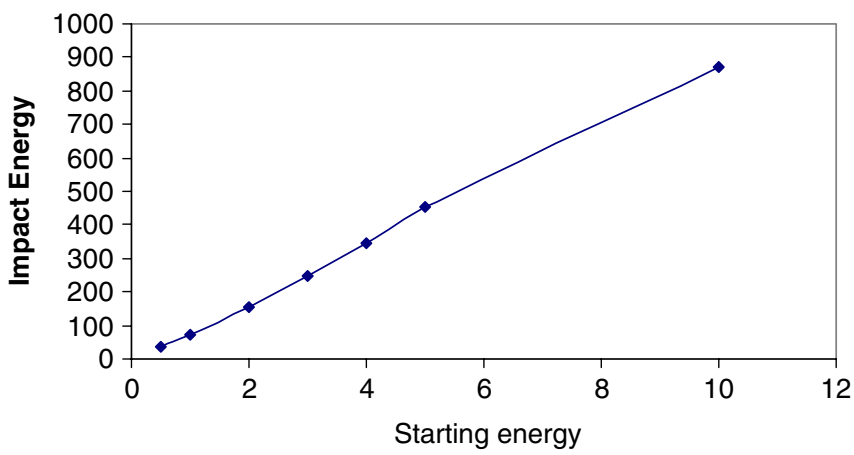

FIG. 11. Impact energy as a function of electron starting energy. 


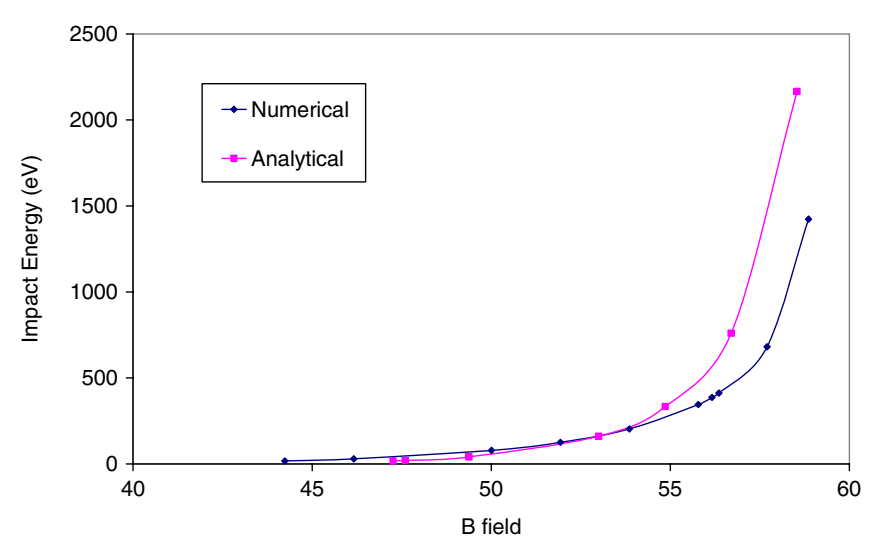

FIG. 12. Comparison between the numerically calculated impact energy and the analytically predicted, for multipactor in a rf deflecting cavity with an iris of $70 \mathrm{~mm}$ radius.

cycle. Eventually the radius will become so large that the fields are no longer correct to keep synchronism and the macroparticle will quickly lose charge. In tracking codes where the focus is on the number of electrons after a fixed time, as we will use later, this may appear as multipactor if the simulation is not run for long enough to see the electrons lose synchronism.

\section{COMPARISON WITH KEKB CRAB CAVITY}

The KEKB crab cavity operates at $508 \mathrm{MHz}$ and has two beam pipes with diameters of 188 and $240 \mathrm{~mm}$. Multipactor was found to occur in the smaller beam pipe by heat rises and x-ray emission at a peak surface electric fields of 12 and $18 \mathrm{MV} / \mathrm{m}$. It is assumed that the $18 \mathrm{MV} / \mathrm{m}$ multipactor is a two-point first order [11]. At this gradient the peak magnetic field on the iris is $37.5 \mathrm{mT}$. As the beam pipe is rounded the magnetic field on the iris at a surface electric field of $18 \mathrm{MV} / \mathrm{m}$ varies between 20.8 and $37.5 \mathrm{mT}$. The analytical predictions from Eq. (24) indicate multipactor starting at $28.5 \mathrm{mT}$ at a launch phase of 0 degrees. However, the impact energy at this phase is equal to the emission energy of a few $\mathrm{eV}$, hence a higher starting phase and magnetic field is more likely as these would provide an impact energy above the threshold energy for multipactor, $\sim 40 \mathrm{eV}$. At a launch phase of 22 degrees, the impact energy is $40 \mathrm{eV}$ and the predicted magnetic field is $31 \mathrm{mT}$. Using an average electron emission energy of $4 \mathrm{eV}$, the equations in this paper predict multipactor to occur at magnetic fields of 30 to $36 \mathrm{mT}$, which is in excellent agreement with the experimental observations.

\section{SECOND ORDER MULTIPACTOR}

As mentioned previously higher order multipactor is also possible, where the electrons take multiples of half the rf period between surface impacts. These multipacting trajectories occur at lower field levels and have a smaller phase focusing range than the first order multipactors. One higher order trajectory of concern is a two-point multipactor that occurs over $1.5 \mathrm{rf}$ cycles. In this case the electrons travel around a large circular orbit in the first half cycle as before; however, due to the lower fields they do not reach the cavity walls in that half period. When the magnetic field changes direction the electron velocity changes also direction due to the electric field, hence making a small orbit. When the magnetic field becomes positive again the electron performs a larger orbit and hits the wall. The stable launch phases for these particles are found to be close to 90 degrees meaning the trajectories start in a large magnetic field pushing them outwards.

These trajectories are not studied analytically here but have been studied using the numerical tracking calculation. An example trajectory is shown in Fig. 13 for a peak magnetic field of $29 \mathrm{mT}$. These trajectories are only found in numerical tracking simulations to have resonant trajectories at $\mathrm{rf}$ magnetic fields of 27 to $31 \mathrm{mT}$ and all have impact energies between 20 and $300 \mathrm{eV}$. The magnetic field at which multipactor occurs is close to that of a single cyclotron orbit in a DC magnetic field, i.e.,

$$
B=\frac{\omega_{\mathrm{rf} m}}{e} .
$$

This equation gives a magnetic field of $28.6 \mathrm{mT}$ for second order multipactor to occur in a cavity with an iris diameter of $70 \mathrm{~mm}$.

If we apply these formulas to the KEKB crab cavity, this multipacting band is predicted to occur at $18.1 \mathrm{mT}$. The experiments performed at KEKB estimate this band to occur over peak magnetic fields between 13.8 and $25.0 \mathrm{mT}$ [11] showing good agreement between the experimental results and the predictions. The ratio of the two magnetic fields at which the two multipactor orders occurred was around 0.66 which is identical to the ratio

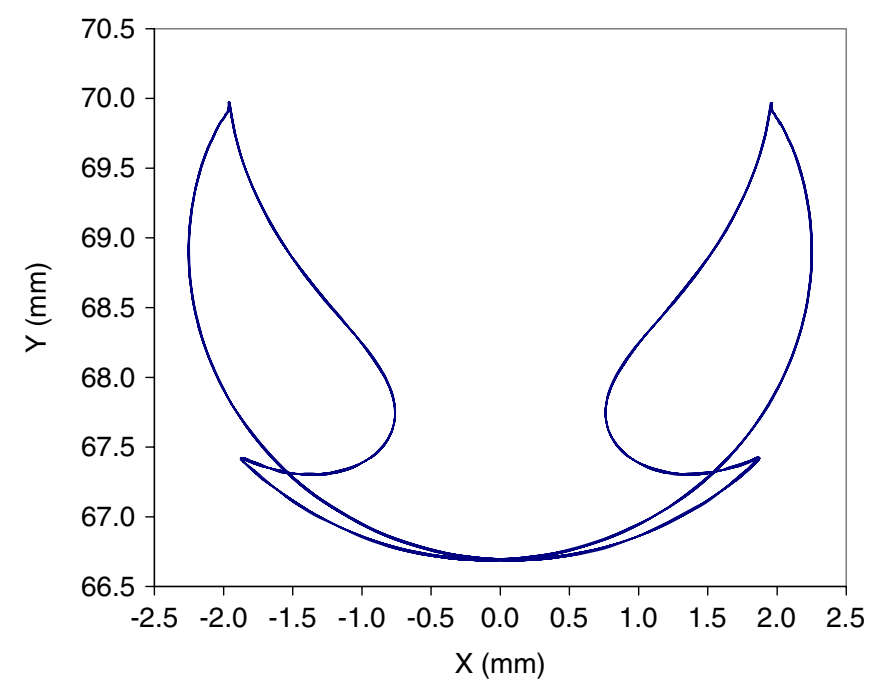

FIG. 13. Second order multipactor trajectory. 
measured at KEKB [11]. However, the $12 \mathrm{MV} / \mathrm{m}$ multipactor band at KEKB was suspected to be a one-point multipactor over one rf period; however, the simulations performed here seem to indicate the two-point MP is more likely.

The SPX crab cavity has reported multipactor at around $90 \mathrm{mT}$ in their $2.8 \mathrm{GHz}$ prototype cavity [18]. This is only marginally lower than the $100 \mathrm{mT}$ predicted by (34) suggesting this is a second order single point multipactor in the cavity iris.

\section{NUMERICAL SIMULATIONS OF CRAB CAVITIES}

CST PARTICLE STUDIO [13] has previously been used to simulate multipactor in rectangular waveguides [19] and in accelerating cavities [20]. The same methods used are applied here to study the onset of multipactor in deflecting mode cavities. In the previous sections we have numerically calculated the trajectories of electrons in the fields of an $\mathrm{HM}_{11}$ mode. While the fields in a dipole cavity will be similar to this, they will contain small components of other modes; hence, it is necessary to simulate the multipactor is a full 3D code with the ability to simulate arbitrary geometries. The cavity fields are initially calculated using the eigenmode solver in CST STUDIO suite. A very dense mesh of around 70 lines per wavelength is used in order to obtain accurate fields close to the walls. The tracking solver in CST PARTICLE STUDIO is then used to track electrons emitted from the cavity iris in the calculated fields at a given phase and amplitude. When an electron impacts a surface, the SEY model specified by the user is used to determine the number of secondaries produced, their emission angle, and their emission energy. The statistical method used by Furman and Pivi [2] is used here and, hence, elastic and inelastic collisions are also possible.

To determine if multipactor is likely, the total number of electron impacts on the cavity walls and the total number of secondaries produced by these impacts are recorded. By dividing the total number of secondaries produced by the total number of electron impacts we are able to define an average secondary emission yield, $\langle\mathrm{SEY}\rangle$.

$$
\begin{aligned}
\langle\mathrm{SEY}\rangle= & (\text { total number of secondaries produced }) / \\
& \text { (total number of impacts). }
\end{aligned}
$$

The number of electrons, $N_{e}$, increases as $N_{e}=\langle\mathrm{SEY}\rangle^{n}$, where $n$ is the number of impacts/rf cycles, hence $\langle\mathrm{SEY}\rangle$ is a measure of the growth of the number of electrons independent of time. If $\langle\mathrm{SEY}\rangle$ is above 1.0 then multipactor is possible, the higher $\langle\mathrm{SEY}\rangle$ the higher the probability of multipactor occurring as the total number of electrons will grow sharply. However, a $\langle\mathrm{SEY}\rangle$ close to 1.0 is unlikely to progress to multipactor as the growth rate is too slow to reach an appreciable current.

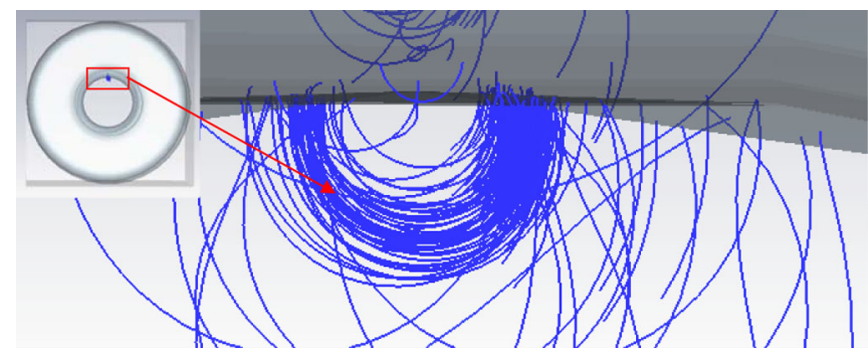

FIG. 14. Electron trajectories from CST PARTICLE STUDIO. The left inset shows the position of the multipactor on the iris.

The exact SEY of niobium in not well studied and exact probability functions for true secondaries, rediffused electrons, and reflected electrons are not known. In the calculations the SEY of niobium is specified by using the Furman-Pivi statistics for copper and modifying the energy at which peak multipactor occurs and the peak SEY to those specified in [15] with the peak yield of 1.5 occurring at an impact energy of $250 \mathrm{eV}$. The tracking simulations are run over a number of launch phases and the maximum $\langle\mathrm{SEY}\rangle$ over all phases is recorded. This is repeated over a number of field amplitudes to study the level of multipactor at various cavity voltages.

The onset of multipactor was investigated as a function of cavity fields for several cavity geometries all with resonant frequencies of $800 \mathrm{MHz}$. The iris radius was varied between 50 and $70 \mathrm{~mm}$ and the rounding on the iris was varied between 30 and $60 \mathrm{~mm}$. The semicircular trajectories predicted in the previous sections can clearly be seen in Fig. 14. When we look at the peak level of multipactor, we can see each cavity shows the onset and peaks of multipactor at different voltage levels, shown in Fig. 15.

However, when we look at multipactor as a function of peak surface magnetic field we find the cavities all show peak multipactor at roughly the same magnetic field as predicted. The average SEY as a function of peak magnetic

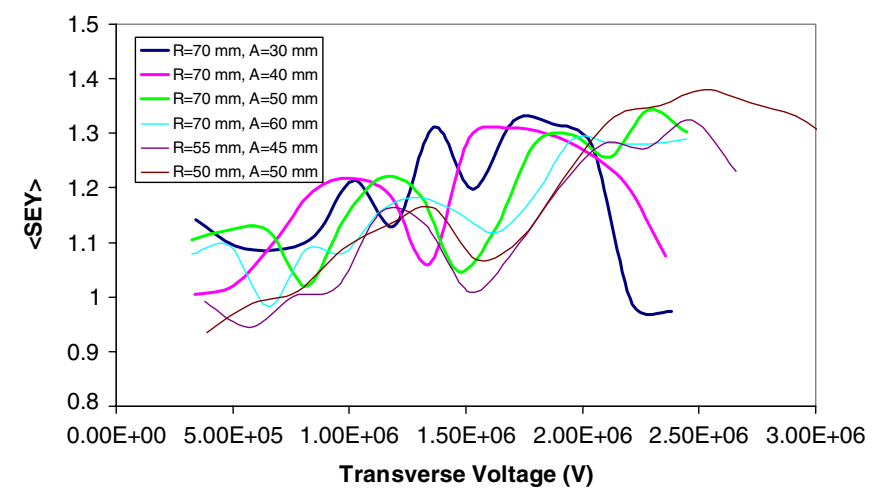

FIG. 15. Maximum $\langle\mathrm{SEY}\rangle$; as a function of transverse voltage from CST PARTICLE STUDIO for various $800 \mathrm{MHz}$ cavity geometries. The iris radius, $R$, and the iris rounding, $A$, are given for each geometry. 


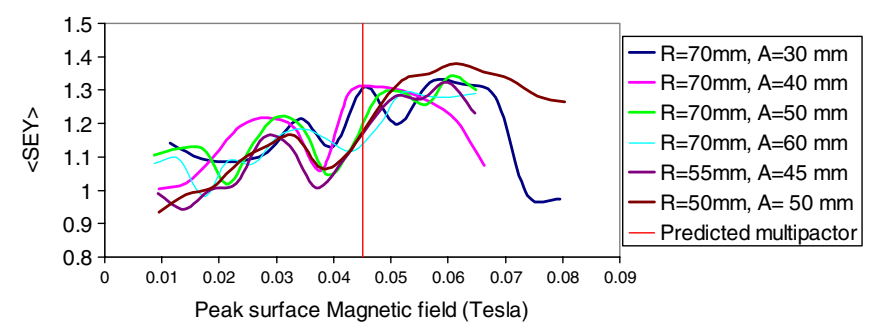

FIG. 16. Maximum $\langle\mathrm{SEY}\rangle$ as a function of peak surface magnetic field from CST PARTICLE STUDIO for various $800 \mathrm{MHz}$ cavity geometries. The iris radius, $R$, and the iris rounding, $A$, are given for each geometry. The red line shows the predicted onset of multipactor.

field is shown in Fig. 16. The first order multipactor is found to start at a peak magnetic field of around $40 \mathrm{mT}$ but is not fully established until the magnetic field reaches around $45-50 \mathrm{mT}$. The spread in starting magnetic field is likely to be due to the difference in impact energy for different geometries. Equation (24) predicts the onset of multipactor at a magnetic field of $45 \mathrm{mT}$ which is in good agreement with the CST simulations.

The second order multipactor can also be seen in Fig. 16 at a peak surface magnetic field of around $30 \mathrm{mT}$. The two bands seem to slightly overlap. Equation (34) predicts this to start at $29 \mathrm{mT}$ which is also in excellent agreement with the CST results.

\section{CONCLUSION}

Multipactor in the iris of dipole cavities has been investigated using analytical techniques, numerical particle tracking in analytical fields, and CST PARTICLE STUDIO. Excellent agreement between all three has been shown. The analytical functions derived in this paper allow rapid prediction of multipactor in dipole cavities and allow an understanding of the physics of the resonant trajectories. This should enable cavity designers to utilize these simple equations at an early stage in order to avoid multipactor before resorting to extensive numerical tracking simulations.

The equations contained in this paper show that dipole cavities are likely to multipact when the peak magnetic field in the iris reaches a certain limit given by the frequency of the cavity. Avoiding such multipactor can be achieved by either reducing the peak magnetic field in the iris or modifying the iris geometry so that the fields in the iris are perturbed.
It has been shown that the multipactor is dependent mainly on the peak surface magnetic field on the iris, and the frequency of operation. This means that these results are also relevant to the novel dipole cavities being developed for LHC [21] as well as traditional $\mathrm{TM}_{110}$ like cavities. As the magnetic field is limited in superconducting cavities to around $200 \mathrm{mT}$ an upper frequency limit of $1.78 \mathrm{GHz}$ is placed on first order multipactor of this type; however second order, or higher, multipactor may still be possible in cavities operating at frequencies above this limit.

[1] R. M. Vaughn, IEEE Trans. Electron Devices 35, 1172 (1988).

[2] M. A. Furman and M. T.F. Pivi, Phys. Rev. ST Accel. Beams 5, 124404 (2002).

[3] S. Riyopoulos, Phys. Plasmas 4, 1448 (1997).

[4] A.C. Dexter and R. Seviour, in the 4th International Workshop on Multipactor, Corona and Passive Intermodulation in Space RF Hardware, 2003, Noordwijk.

[5] V.E. Semenov et al., Phys. Plasmas 17, 123503 (2010).

[6] R. Udiljak et al., Phys. Plasmas 14, 033508 (2007).

[7] P. Yla-Oijala, Part. Accel. 63, 105 (1999).

[8] R. B. Palmer, Report No. SLAC-PUB-4707, 1988.

[9] M. McAshan and R. Wanzenberg, Report No. FERMILAB-TM-2144, 2001.

[10] H. Hahn, Rev. Sci. Instrum. 34, 10 (1963).

[11] Y. Morita et al., in Proceedings of SRF03, Lubeck, 2003, TuP23.

[12] L. Xiao et al., in Proceedings of the 23rd Particle Accelerator Conference, Vancouver, Canada, 2009 (IEEE, Piscataway, NJ, 2009), WE5PFP048.

[13] CST, http://www.cst.com/.

[14] Y. Garault, CERN Report No. 64-43, 1964.

[15] R. Calder, G. Dominichini, and N. Hilleret, Report No. CERN-LEP-VA/87-61, 1987.

[16] J. C. Slater, Electromagnetism (Dover, New York, 1969), Chap. XIII, 1st ed.

[17] C. Adophsen et al., EuroTeV-report-2007-010/ FERMILAB-TM-2388, 2007.

[18] H. Wang, Four Rod LHC Crab Cavity Workshop (Cockcroft Institute, UK, 2011).

[19] G. Burt et al., in Proceedings of SRF 09, Berlin, TUPP046.

[20] G. Romanov, in LINAC08, Victoria (2008), MOP043.

[21] G. Burt, New Cavity Shape Developments for Crabbing Applications, SRF 09, Berlin. 